\title{
CHOLANGIOCARCINOMA MISCODING IN HEPATOBILIARY CENTRES
}

Shaun Selvadurai ${ }^{1}$, Kulbir Mann ${ }^{2}$, Sanjena Mithra ${ }^{3}$, John Bridgewater ${ }^{3,4}$, Hassan Malik ${ }^{2}$, Shahid A Khan $^{1}$

${ }^{1}$ Liver Unit, Division of Digestive Diseases, Imperial College London and Imperial College Healthcare NHS Trust

${ }^{2}$ Aintree University Hospital Foundation Trust

${ }^{3}$ University College London Hospitals NHS Trust, London

${ }^{4}$ UCL Cancer Institute, London

Corresponding Author: Shahid A Khan, Liver Unit, 10 ${ }^{\text {th }}$ Floor QEQM Building St Mary's Hospital, South Wharf Road, London W2 1NY, UK

Email: shahid.khan@imperial.ac.uk

Tel: +442033126454

Fax: +44 2074022796

Word Count: 2239

Figures: 2

Tables: 4

List of abbreviations in order of Appearance:

Cholangiocarcinoma (CCA), Intrahepatic Cholangiocarcinoma (iCCA), Extrahepatic

Cholangiocarcinoma (eCCA), perihilar cholangiocarcinoma (pCCA), distal cholangiocarcinoma (dCCA), Imperial College Healthcare NHS Trust (ICHT), University College London Hospitals NHS Foundation Trust (UCHT) and Aintree University Hospital NHS Foundation Trust (AUHT), C22.1 Intrahepatic Bile Duct carcinoma (C22.1 iCCA), C24.0 Extrahepatic Bile Duct carcinoma (c24.0 eCCA), C23X Malignant Neoplasm Gall Bladder (C23X GB), C22.0 Malignant Neoplasm Liver Cell Carcinoma (C22 HCC), C24.1 Ampullary Malignancy (C24.1 AMP), HepatoPancreatoBiliary (HPB), United Kingdom (UK)

Conflict of Interest and Disclosures: The authors have no pertinent financial support or conflicts of interest to declare.

Acknowledgements: JB and SK are grateful for support from the United Kingdom National Institutes for Health Research (NIHR) Biomedical Facilities at UCL and Imperial College London, respectively. 
ABSTRACT (248 words)

\section{Introduction}

Cholangiocarcinoma (CCA) are sub-divided into intrahepatic (iCCA) or extrahepatic (eCCA). eCCA are further subdivided into perihilar (pCCA) and distal (dCCA). Current and previous versions of the WHO International Coding of Disease and Oncology classifications (ICD) have separate topography codes for iCCA and eCCA, but none for pCCA. Over recent decades, multiple studies report rising incidence rates of iCCA with declining rates of eCCA, without reference to pCCA. We hypothesised the lack of a specific code for pCCA has led to errors CCA coding, specifically with miscoding of pCCA as iCCA.

\section{Methods}

Clinical notes of cases coded as hepatobiliary carcinoma using ICD-10 criteria (C22.1/Intrahepatic Bile Duct carcinoma, C24.0/Extrahepatic Bile Duct carcinoma, C23X/Malignant Neoplasm Gall Bladder, C22.0/Malignant Neoplasm Liver Cell Carcinoma) over a 2 year period (2015-2017), were reviewed by two independent clinicians at three independent UK regional HepatoPancreatoBiliary centres. The agreed final diagnosis was compared to the originally allocated ICD-10 code.

\section{Results}

Of the 625 CCA cases fully reviewed, 226 were coded as C22.1/iCCA. 98 (43\%) of these were true iCCA and coded correctly, while 76 cases (34\%) were actually pCCA. 92\% all pCCA cases were incorrectly coded as iCCA.

\section{Conclusion}

CCA coding misclassification in UK HPB centres is common, particularly the miscoding of pCCA, which is extrahepatic and the commonest form of CCA, as iCCA. This may be contributing to apparent rising incidence rates of iCCA. Our findings confirm the need to implement distinct topographical codes for iCCA, pCCA and dCCA in future iterations of ICD. 


\section{INTRODUCTION}

Cholangiocarcinoma (CCA) constitutes a group of epithelial malignancies arising from the biliary tree. They are the most common biliary malignancy, and second most common hepatobiliary malignancy, accounting for $10-15 \%$ of the total incidence [1][2]. CCA present insidiously with minimal symptoms in the early course of disease, often without any known risk factors, and are therefore associated with a late diagnosis and high mortality.

CCA are classified based on their anatomical location within the biliary tree. Intrahepatic Cholangiocarcinoma (iCCA) arise from biliary ducts within the hepatic parenchyma, anatomically above the second-order bile ducts [3]. iCCA are the second commonest primary liver malignancy after hepatocellular carcinoma (HCC) [3-9]. Extrahepatic cholangiocarcinoma (eCCA) arise distal to the origin of the second order biliary ducts and are sub-divided into perihilar cholangiocarcinoma (pCCA, historically loosely termed "Klatskin tumours") and distal cholangiocarcinoma (dCCA). dCCA originates between the cystic duct and ampulla of Vater, thus the cystic duct is the anatomical point of distinction between pCCA and dCCAs [3]. Each of the three sub-types have a distinct epidemiology, pathophysiology, prognosis and approach to clinical management $[3,16]$. In clinical practice and published data, pCCA is the most common sub-type of CCA, accounting for $50-60 \%$ of CCA with the minority being iCCA or dCCA (other than in Asia and mainland Europe where iCCA is the predominant subtype) $[3,4,20-23]$.

Several studies over the past few decades have reported an increasing incidence in CCA globally. Using multiple data bases, including the World Health Organisation (WHO) Database [5], US cancer registry, $[6,7,8]$ Japanese and other European registries $[9,10,11]$, these studies have consistently found rising incidence of iCCA, whilst the incidence of eCCA has remained relatively stable or decreased [12, 15].

The WHO classifies all medical diagnoses utilising the International Classification of Diseases (ICD-10) coding system. ICD-10, and its previous iterations, has separate topography codes for iCCA and eCCA, however omits a code for PCCA. In parallel, a separate coding system just for cancers is produced by the International Agency of Research on Cancer (IARC), the specialized cancer agency of the WHO. This is the International Classification of Diseases for Oncology (ICD-0), which consists of two coding sub-systems, which together describe the tumour: the topographical code, which describes the anatomical site of origin of the tumour; and the morphological code, which describes the cell type (or histology) of the tumour, together with the behaviour (malignant or benign). ICD-O does give "Klatskin" CCA a unique histology code, 8162/3, but previous iterations of ICD-0 (ICD-02), incorrectly cross referenced pCCA tumour morphology with iCCA topography. The version currently in use (ICD03) cross references pCCA tumour morphology with either iCCA or eCCA topography. A study of the 
US surveillance, epidemiology and end results database found, following the introduction of ICD-03 in 2001, the incidence of iCCA reduced from 0.91 per 100,00 in 2001, to 0.6 per 100,000 by 2007 . Conversely the incidence rate eCCA increased from 0.8 per 100,000 in 2001 to 0.97 per 100,000 in 2007, with a large proportion of pCCA tumours now being cross-referenced as eCCA. [13] This discrepancy demonstrates the significance of inconsistent and incorrect coding for epidemiological studies. The lack of accurate coding available for pCCA raises questions as to the true epidemiology of CCA subtypes.

We hypothesised that misclassification of CCA coding is common, which could skew the incidence of CCA coded as iCCA. The aims of this study were to retrospectively analyse case notes of patients coded with the major hepatobiliary malignancies at multiple regional HepatoPancreatoBiliary (HPB) centres to accurately reflect the prevalence of CCA subtypes.

\section{METHODS}

A retrospective clinical review of cases coded as hepatobiliary carcinoma using ICD-10 criteria was performed at three regional UK HPB Centres: Imperial College Healthcare NHS Trust (ICHT) covering West and North West London, University College London Hospitals NHS Foundation Trust (UCHT) covering North London and Aintree University Hospital NHS Foundation Trust (AUHT) covering the Merseyside region. The following diagnostic codes of the major hepatobiliary cancers from hospital records were reviewed: C22.1 Intrahepatic Bile Duct carcinoma (C22.1/iCCA), C24.0 Extrahepatic Bile Duct carcinoma (C24.0/eCCA), C23X Malignant Neoplasm Gall Bladder (C23X/GB), C22.0/Malignant Neoplasm Liver Cell Carcinoma (HCC) and C24.1/Ampullary Malignancy (AMP). The study period was $1^{\text {st }} J a n 2015$ to $1^{\text {st }}$ Jan 2017. Patient's clinical data including clinic letters, outpatient and inpatient notes, histology, imaging and multidisciplinary team outcomes, were reviewed to ascertain the exact sub-type of CCA by two independent clinicians at each regional centre. Patients were excluded if there was limited clinical information. Data was collated, analysed and visual representations of the data were made using Microsoft Excel 2013.

\section{RESULTS}

A total of 744 CCA cases were assessed from across all three centres. 119 were excluded due to clinical data being insufficient to confidently confirm the diagnosis and/or location of the tumour. Of the 625 cases that were fully reviewed, 226 cases were coded as C22.1/iCCA, of which 98 cases (43\%) were deemed to have been correctly coded. 17 cases were coded as C24.0/eCCA, of which 9 cases (53\%) were correctly coded. 81 cases were coded as C23X/GB, of which 73 cases (90\%) were correctly coded. 296 cases were coded as C22/HCC, of which 288 cases (97\%) were correctly coded. 5 cases were coded as C24.1/AMP, of which 3 cases $(60 \%)$ were correctly coded.(Table 1 ) The accuracy of coding as 
C22.1/iCCA was low across all centres: $42 \%, 26 \%$ and $47 \%$ of cases were correctly coded at ICHT, UCHT and AUHT respectively (Tables $2 \mathrm{a}, \mathrm{b}, \mathrm{c})$.

\begin{tabular}{|l|l|l|l|l|}
\hline Code & $\begin{array}{l}\text { Total Cases } \\
\text { across all sites }\end{array}$ & $\begin{array}{l}\text { Cases Fully } \\
\text { Reviewed }\end{array}$ & $\begin{array}{l}\text { Cases Correctly } \\
\text { Coded }\end{array}$ & $\begin{array}{l}\text { Percentage } \\
\text { Coded Correctly }\end{array}$ \\
\hline C22.1 IHBD CARCINOMA & 233 & 226 & 98 & $43 \%$ \\
\hline C24.0 EHBD CARCINOMA & 18 & 17 & 9 & $53 \%$ \\
\hline $\begin{array}{l}\text { C23X MALIGNANT NEOPLASM } \\
\text { GALL BLADDER }\end{array}$ & 110 & 81 & 73 & $90 \%$ \\
\hline $\begin{array}{l}\text { C22.0 MALIGNANT NEOPLASM } \\
\text { LIVER CELL CARCINOMA }\end{array}$ & 378 & 296 & 288 & $97 \%$ \\
\hline C24.1 AMPULLARY MALIGNANCY & 5 & 5 & 3 & $60 \%$ \\
\hline
\end{tabular}

Table 1: Proportion of Cases reviewed and Coded Correctly across sites

\begin{tabular}{|l|l|l|l|l|}
\hline Code & $\begin{array}{l}\text { Total Cases at } \\
\text { ICHT }\end{array}$ & $\begin{array}{l}\text { Cases Fully } \\
\text { Reviewed }\end{array}$ & $\begin{array}{l}\text { Cases Correctly } \\
\text { Coded }\end{array}$ & $\begin{array}{l}\text { Percentage } \\
\text { Coded Correctly }\end{array}$ \\
\hline C22.1 IHBD CARCINOMA & 80 & 73 & 31 & $42 \%$ \\
\hline C24.0 EHBD CARCINOMA & 4 & 3 & 3 & $100 \%$ \\
\hline $\begin{array}{l}\text { C23X MALIGNANT NEOPLASM } \\
\text { GALL BLADDER }\end{array}$ & 59 & 31 & 27 & $87 \%$ \\
\hline $\begin{array}{l}\text { C22.0 MALIGNANT NEOPLASM } \\
\text { LIVER CELL CARCINOMA }\end{array}$ & 186 & 104 & 100 & $97 \%$ \\
\hline
\end{tabular}

Table 2a) Proportion of Cases reviewed and Coded Correctly at ICHT

\begin{tabular}{|l|l|l|l|l|}
\hline Code & $\begin{array}{l}\text { Total Cases at } \\
\text { UCHT }\end{array}$ & $\begin{array}{l}\text { Cases Fully } \\
\text { Reviewed }\end{array}$ & $\begin{array}{l}\text { Cases Correctly } \\
\text { Coded }\end{array}$ & $\begin{array}{l}\text { Percentage } \\
\text { Coded Correctly }\end{array}$ \\
\hline C22.1 IHBD CARCINOMA & 23 & 23 & 6 & $26 \%$ \\
\hline C24.0 EHBD CARCINOMA & 4 & 4 & 0 & $0 \%$ \\
\hline $\begin{array}{l}\text { C23X MALIGNANT NEOPLASM } \\
\text { GALL BLADDER }\end{array}$ & 10 & 9 & 8 & $89 \%$ \\
\hline C24.1 AMPULLARY MALIGNANCY & 2 & 2 & 0 & $0 \%$ \\
\hline
\end{tabular}

Table 2b) Proportion of Cases reviewed and Coded Correctly at UCHT

\begin{tabular}{|l|l|l|l|l|}
\hline Code & $\begin{array}{l}\text { Total Cases at } \\
\text { AUHT }\end{array}$ & $\begin{array}{l}\text { Cases Fully } \\
\text { Reviewed }\end{array}$ & $\begin{array}{l}\text { Cases Correctly } \\
\text { Coded }\end{array}$ & $\begin{array}{l}\text { Percentage } \\
\text { Coded Correctly }\end{array}$ \\
\hline C22.1 IHBD CARCINOMA & 130 & 130 & 61 & $47 \%$ \\
\hline C24.0 EHBD CARCINOMA & 10 & 10 & 6 & $60 \%$ \\
\hline $\begin{array}{l}\text { C23X MALIGNANT NEOPLASM } \\
\text { GALL BLADDER }\end{array}$ & 41 & 41 & 38 & $93 \%$ \\
\hline $\begin{array}{l}\text { C22.0 MALIGNANT NEOPLASM } \\
\text { LIVER CELL CARCINOMA }\end{array}$ & 192 & 192 & 188 & $98 \%$ \\
\hline C24.1 AMPULLARY MALIGNANCY & 3 & 3 & 3 & $100 \%$ \\
\hline
\end{tabular}

Table 2c) Proportion of Cases reviewed and coded correctly at AUHT 
Of the 73 cases coded as C22.1 iCCA at ICHT, 31 cases (42\%) were true iCCA, 23 cases (32\%) pCCA, 7 cases (10\%) dCCA, 2 cases (3\%) GB, and 10 cases (14\%) were other diagnoses, including hepatic/ duodenal malignancies, metastatic disease or unspecified. Of these cases, 12(38\%) of iCCA cases, 11 $(47 \%)$ of pCCA cases, 6 (86\%) of dCCA cases had an operative intervention during their disease course.

Of the 23 cases coded as C22.1/iCCA at UCHT, 6 cases (26\%) were true iCCA, 15 cases (65\%) pCCA and 2 cases (9\%) were other diagnoses. Of these cases, $3(50 \%)$ of iCCA cases, $5(33 \%)$ of pCCA cases and $2(100 \%)$ of other diagnoses cases had an operative intervention during their disease course.

Of the 130 cases coded as C22.1/iCCA at AUHT 61 (47\%) were true iCCA, 38 (29\%) pCCA, 22 (17\%) dCCA, 4 (3\%) GB, and 5 (4\%) were other diagnoses: hepatic/ duodenal malignancies, metastasis or unspecified. Of these cases, 12(20\%) of iCCA cases, 17 (45\%) of pCCA cases, 16 (73\%) of dCCA cases, 3 (75\%) GB cases and 2 (40\%) of other diagnosis cases had an operative intervention during their disease course. Cases which had no operative intervention was either because the patient was deemed unfit for surgery, the tumour was deemed to be inoperable, or the patient declined surgery. (Table 3a,Table 3b) Figure 1).

\begin{tabular}{|c|c|c|c|}
\hline & Number ( & of Cases b & \\
\hline $\begin{array}{l}\text { Actual Diagnosis for Patients coded } \\
\text { as C22.1 IHBD CARCINOMA }\end{array}$ & ICHT & UCHT & AUHT \\
\hline $\begin{array}{l}\text { True Intrahepatic } \\
\text { Cholangiocarcinoma (iCCA) }\end{array}$ & $31(42 \%)$ & $6(26 \%)$ & $61(47 \%)$ \\
\hline Perihilar Cholangiocarcinoma (pCCA) & $23(32 \%)$ & $15(65 \%)$ & $38(29 \%)$ \\
\hline Distal Cholangiocarcinoma (dCCA) & $7(10 \%)$ & 0 & $22(17 \%)$ \\
\hline Gallbladder Carcinoma (GB) & $2(3 \%)$ & 0 & $4(3 \%)$ \\
\hline $\begin{array}{l}\text { Other (Hepatic, Dudodenal } \\
\text { Carcinoma, Metastatic disease, } \\
\text { Unspecified) }\end{array}$ & $10(14 \%)$ & $2(9 \%)$ & $5(4 \%)$ \\
\hline
\end{tabular}

Table 3a: Actual Diagnosis for Patients coded as C22.1 IHBD CARCINOMA by Centre

\begin{tabular}{|c|c|c|c|}
\hline \multirow[b]{2}{*}{$\begin{array}{l}\text { Actual Diagnosis for Patients coded } \\
\text { as C22.1 IHBD CARCINOMA }\end{array}$} & \multicolumn{3}{|c|}{$\begin{array}{l}\text { Number (Percentage) of Cases which had operative intervention by } \\
\text { Centre }\end{array}$} \\
\hline & ICHT & UCHT & AUHT \\
\hline $\begin{array}{l}\text { True Intrahepatic } \\
\text { Cholangiocarcinoma (iCCA) }\end{array}$ & $12(38 \%)$ & $3(50 \%)$ & $12(20 \%)$ \\
\hline Perihilar Cholangiocarcinoma (pCCA) & $11(47 \%)$ & $5(33 \%)$ & $17(45 \%)$ \\
\hline Distal Cholangiocarcinoma (dCCA) & $6(86 \%)$ & 0 & $16(73 \%)$ \\
\hline Gallbladder Carcinoma (GB) & 0 & 0 & $3(75 \%)$ \\
\hline $\begin{array}{l}\text { Other (Hepatic, Dudodenal } \\
\text { Carcinoma, Metastatic disease, } \\
\text { Unspecified) }\end{array}$ & 0 & $2(100 \%)$ & $2(40 \%)$ \\
\hline
\end{tabular}

Table 3b: Cases coded as C22.1IHBD Carcinoma that had operative intervention 


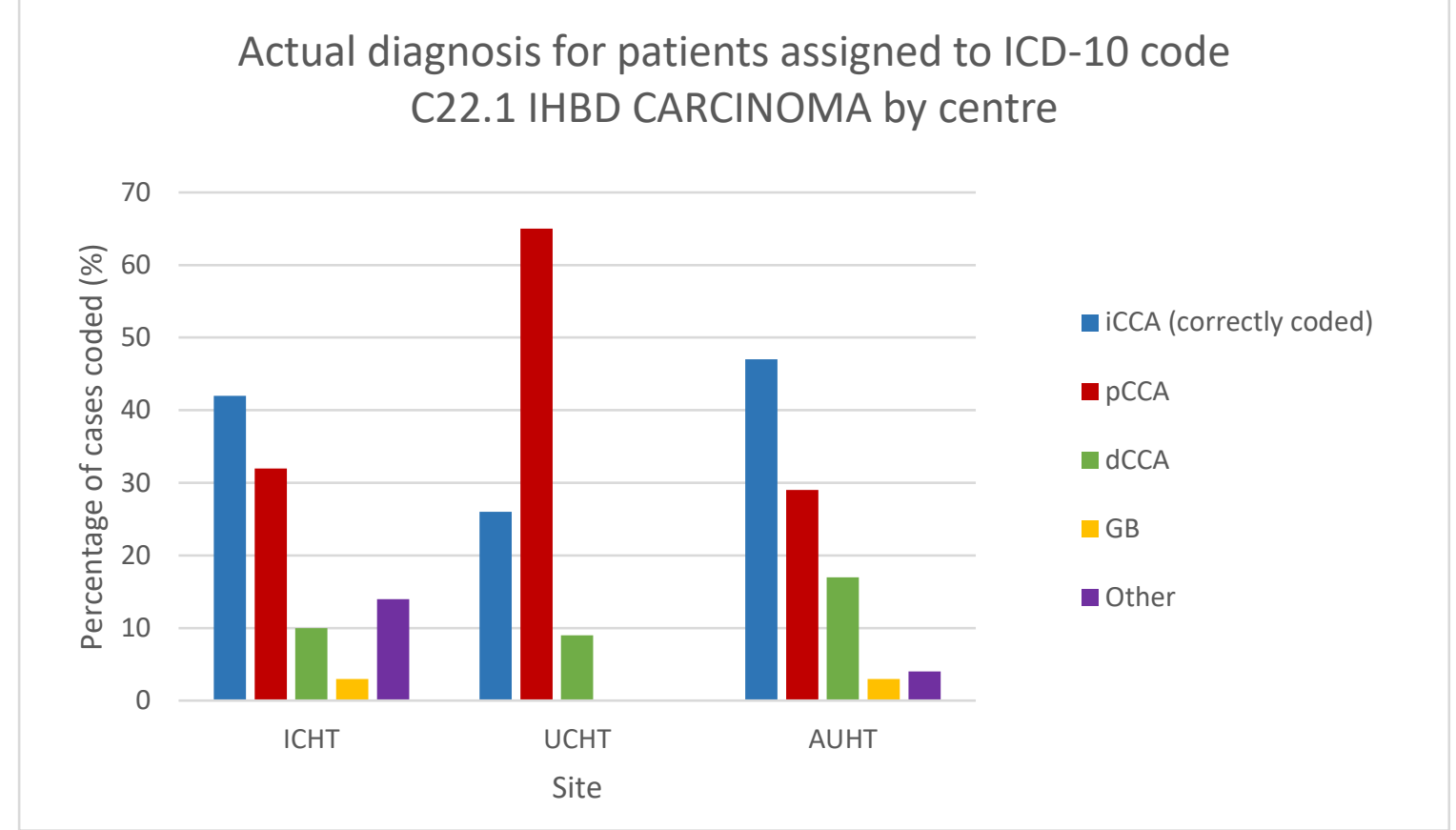

Figure 1: Actual Diagnosis for Patients ICD-10 code C22.1 IHBD CARCINOMA by Centre

Of the 226 cases coded as C22.1/iCCA across all centres, 98 cases (43\%) were coded correctly as iCCA, leaving more than half incorrectly coded. 76 cases (34\%) were actually pCCA, 29 cases (13\%) were dCCA, 6 cases (3\%) were GB, 17 cases (7\%) were other diagnoses, including hepatic or duodenal carcinoma, metastatic disease, or unspecified.

Of the 27 cases deemed to be true pCCA at ICHT, 23 cases (85\%) were coded as C22.1/iCCA with the remaining 4 cases (15\%) being coded as C23X/GB or unspecified. Of the 16 cases found to be true pCCA at UCHT, 15 cases (94\%) were coded as C22.1/iCCA, with the remaining 1 case (6\%) coded as unspecified. Of the 40 cases coded at AUHT, 38 cases (95\%) were coded as C22.1/iCCA, with the remaining 2 cases (5\%) being coded as C24.0/eCCA (Table 4)(Figure 2).

\begin{tabular}{|l|l|l|l|}
\hline & \multicolumn{3}{|l|}{ Number (Percentage) of Cases Coded by Centre } \\
\hline $\begin{array}{l}\text { Code given to Cases of Perihilar } \\
\text { Cholangiocarcinoma }\end{array}$ & ICHT & UCHT & AUHT \\
\hline C22.1 IHBD CARCINOMA & $23(85 \%)$ & $15(94 \%)$ & $38(95 \%)$ \\
\hline C24.0 EHBD CARCINOMA & 0 & 0 & $2(5 \%)$ \\
\hline $\begin{array}{l}\text { Other (C22.0 Neoplasm Liver } \\
\text { Cell,C23X Neoplasm of Gallbladder, } \\
\text { C22 Neoplasm of Liver unspecified) }\end{array}$ & $4(15 \%)$ & $1(6 \%)$ & 0 \\
\hline
\end{tabular}

Table 4: Code given to Cases of Perihilar Cholangiocarcinoma by Centre 


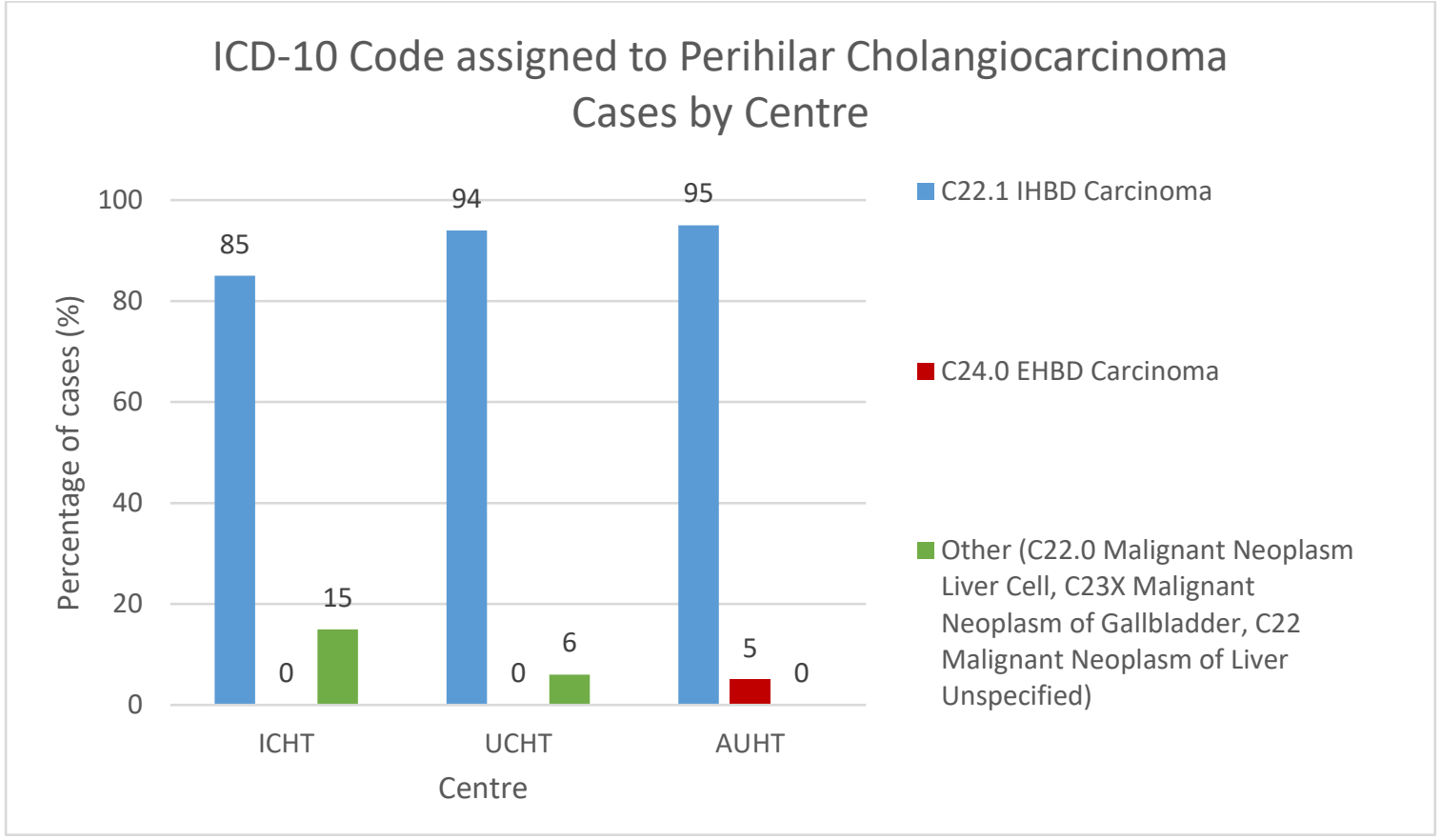

Figure 2: ICD-10 Code assigned to Perihilar Cholangiocarcinoma Cases by Centre

Across all centres, of the 83 cases that were deemed to be truly pCCA, 76 cases (92\%) were coded as C22.1/iCCA, 2 cases (2\%) were coded as C24.0/eCCA, and 5 cases (6\%) were coded as other diagnoses, including HCC, GB and unspecified.

\section{DISCUSSION}

Our study confirmed widespread misclassification of CCA coding in a large patient population across three independent HPB centres both inside and out of London: over $90 \%$ of cases verified as pCCA were incorrectly coded as C22.1/iCCA. The lack of a specific ICD topographical code for PCCA has resulted in it being consistently incorrectly coded as iCCA, even though eCCA would be more accurate. This highlights a systemic fault with coding practices as the lack of appropriate codes inevitably creates uncertainty for those who assign them and in the long term could potentially skew reported epidemiological data.

The three centres included in our study cover a geographically large area of the UK and our findings are therefore a reasonable representation of likely coding practices nationally. The pattern of rates of iCCA and eCCA in the UK are similar to those reported in other countries $[2,5,6-12,14,15]$. Given that all these studies refer to the same WHO/ICD codes, it is certainly feasible that the miscoding issues we report are a global phenomenon. Our findings call into question whether the rising incidence rates for iCCA and falling incidence rates for eCCA reported over the past few decades are accurate, or if this phenomenon is, at least partly, a reflection of the incorrect coding of pCCA. 
Every trust in the United Kingdom has a clinical coding department which is responsible for not only the correct allocation of diagnoses and procedures but the allocation of payment for each department. There have been published studies that recognise inaccuracies across all specialties and recommend increased clinician involvement in correcting these errors and ensure appropriate remuneration [16-18]. With respect to the operation itself, an iCCA may require only a liver resection whereas a pCCA would include a common bile duct excision, lymphadenectomy, hepaticojejunostomy and a possible vascular resection in addition. This has significant implications not only perioperatively but for post-operative care and treatment of complications [17]. From a financial perspective there is also a significant difference in iCCA and pCCA, stressing the importance of accurate coding for the hospital $[17,18]$. From our data, although the total numbers are small, the overall trend suggests less operative intervention for iCCA compared to pCCA or dCCA, suggesting more advanced stage disease at diagnosis.

Our study has limitations: it is a retrospective review of case notes which cannot always reflect the nuances of discussions at a cancer board review, where such cases are discussed. Even after a careful multi-disciplinary review, some CCA cases are impossible to definitively code to the correct site of the tumour, for example large tumours involving both the hilum and the deeper liver parenchyma. Furthermore, the mechanisms of coding and level of staffing responsible are not consistent at all sites. Nonetheless, a common theme of misclassification of pCCA has emerged.

In conclusion, there is a need for ICD-11 and subsequent iterations of ICD-0 to have separate morphological and topographical codes for iCCA, pCCA and dCCA. These three types of CCA are anatomically distinct, have differing epidemiology, pathobiology, clinical presentations and management [3, 21-23]. Clearly defined codes for each will help avoid confusion and may allow more accurate and meaningful epidemiological data to be gathered regarding CCA. Until then, reported cholangiocarcinoma/biliary tract cancer epidemiological trends need to be interpreted carefully. However, having an accurate, fit for purpose coding system is not the full answer. Diagnostic data needs to be recorded uniformly and accurately at all levels. The responsibility to do so lies with clinicians, administrators and cancer registries, as well as the international bodies charged with maintaining databases. We need to create awareness of the widespread miscoding of CCA amongst all members of the multi-disciplinary team, at all levels. There should be a particular emphasis on the education of coding personnel, who should have senior clinician back-up to double check the accuracy of coding data, which itself should be regularly audited. Given the recent advances in our understanding of the genetic drivers behind sub-types of CCA, perhaps in the future accurate coding of CCA will also involve molecular profiling. 


\section{References}

[1] Best Pract Res Clin Gastroenterol. 2015 Apr;29(2):221-32. doi: 10.1016/j.bpg.2015.02.003. Epub 2015 Feb 16. https://www.sciencedirect.com/science/article/abs/pii/S1521691815000190 ?via\%3Dihub

[2] Welzel, T.M., McGlynn, K.A., Hsing, A.W. et al. Impact of classification of hilar cholangiocarcinomas (Klatskin tumors) on the incidence of intra- and extrahepatic cholangiocarcinoma in the United States. J Natl Cancer Inst. 2006; 98: 873-875

[3] Rizvi S, Khan SA, Hallemeier CL, Kelley RK, Gores GJ. Cholangiocarcinoma - evolving concepts and therapeutic strategies. Nature Rev Clin Oncol 2017 Oct 10. doi: 10.1038/nrclinonc.2017.157.)

[4] DeOliveira M.L., Cunningham S.C., Cameron, J.L. et al. Cholangiocarcinoma: thirty-one-year experience with 564 patients at a single institution. Ann Surg. 2007; 245: 755-762

[5] Khan SA, Taylor-Robinson SD, Toledano MB, Beck A, Elliott P, Thomas HC: Changing international trends in mortality rates for liver, biliary and pancreatic tumours. J Hepatol 2002;37:806-813.

[6] Patel T: Increasing incidence and mortality of primary intrahepatic cholangiocarcinoma in the United States. Hepatology 2001;33:1353-1357.

[7] Shaib Y, El-Serag HB: The epidemiology of cholangiocarcinoma. Semin Liver Dis 2004;24:115-125.

[8] Shaib YH, Davila JA, McGlynn K, El-Serag HB: Rising incidence of intrahepatic cholangiocarcinoma

in the United States: a true increase? J Hepatol 2004;40:472-477.

[9] Taylor-Robinson SD, Toledano MB, Arora S, et al: Increase in mortality rates from intrahepatic cholangiocarcinoma in England and Wales 1968-1998. Gut 2001;48:816-820.

[10] Alvaro D, Crocetti E, Ferretti S, Bragazzi MC, Capocaccia R; AISF Cholangiocarcinoma committee: Descriptive epidemiology of cholangiocarcinoma in Italy. Dig Liver Dis 2010;42:490-4

[11] Utada M, Ohno Y, Tamaki T, Sobue T, Endo G: Long-term trends in incidence and mortality of intrahepatic and extrahepatic bile duct cancer in Japan. J Epidemiol 2014;24:193-199.

[12] Kristein M.M, Vogel A : Epidemiology and Risk Factors of Cholangiocacinoma. Visc med 2016; 32:395-400 DOI: 10.1159/000453013

[13] 7. Khan, S.A., Emadossadaty, S., Ladep, N.G. et al. Rising trends in cholangiocarcinoma: is the ICD classification system misleading us?. J Hepatol. 2012;56: 848-854

[14] Walter D, Ferstly P et al; Cholangiocarcinoma in Germany: Epidemiologic trends and impact of misclassification. Liver int, 2019, Feb 39(2):316-323 DOI 10.1111/liv

[15] Bertuccio $P$ et al. Global trends in mortality from intrahepatic and extrahepatic cholangiocarcinoma. J Hepatol. 2019 Mar 23. pii: S0168-8278(19)30183-7. doi:

10.1016/j.jhep.2019.03.013. 
[16] Mahbubani K, Georgiades F, Goh EL et al; Clinician-directed improvement in the accuracy of hospital clincal coding. Future Healthc J. 2018 Feb;5(1):47-51. doi: 10.7861/futurehosp.5-1-47.

[17] Heywood NA, Gill MD, Charlwood N et al, Improving accuracy of clinical coding in surgery: collaboration is key. J Surg Res. 2016 Aug;204(2):490-495. doi: 10.1016/j.jss.2016.05.023. Epub 2016 May 24.

[18] Tsopra R, Peckham D, Beirne P et al; The impact of three discharge coding methods on he accuracy of diagnosic coding and hospital reimbursement for inpatient medical care. Int J Med Inform. 2018 Jul;115:35-42. doi: 10.1016/j.ijmedinf.2018.03.015. Epub 2018 Mar 27.

[19] Soares KC, Kamel I, Cosgrove DP, Herman JM, Pawlik TM. Hilar cholangiocarcinoma: diagnosis, treatment options, and management. Hepatobiliary Surg Nutr. 2014; 3(1): 18-34.doi: 10.3978/j.issn.2304-3881.2014.02.05; PMCID: PMC3955000.

[20] Connell LC, Harding JJ, Shia J, Abou-Alfa GK. Combined intrahepatic cholangiocarcinoma and hepatocellular carcinoma. Chin Clin Oncol. 2016 Oct;5(5):66. doi: 10.21037/cco.2016.10.02.

[21] Khan AS, Dageforde LA. Cholangiocarcinoma. Surg Clin North Am. 2019 Apr;99(2):315-335. doi: 10.1016/j.suc.2018.12.004.

[22] Razumilava N, Gores GJ. Cholangiocarcinoma. Lancet. 2014 Jun 21;383(9935):2168-79. doi: 10.1016/S0140-6736(13)61903-0.

[23] Nakeeb A, Pitt HA, Sohn TA, Coleman J, Abrams RA, Piantadosi S, Hruban RH, Lillemoe KD, Yeo $\mathrm{CJ}$, Cameron JL. Cholangiocarcinoma. A spectrum of intrahepatic, perihilar, and distal tumors. Ann Surg. 1996 Oct;224(4):463-73. 